\title{
Optimal Convergence Analysis for Convection Dominated Diffusion Problems
}

\author{
M. A. Mohamed Ali \\ Mathematics Department, Faculty of Science, Suez Canal University, Ismailia, Egypt \\ Email: mohamedali1961@yahoo.com
}

Received July 6, 2013; revised August 9, 2013; accepted September 1, 2013

Copyright (c) 2013 M. A. Mohamed Ali. This is an open access article distributed under the Creative Commons Attribution License, which permits unrestricted use, distribution, and reproduction in any medium, provided the original work is properly cited.

\begin{abstract}
In classical mixed finite element method, the choice of the finite element approximating spaces is restricted by the imposition of the LBB consistency condition. The method of $\mathrm{H}^{1}$-Galerkin mixed finite element method avoids completely the imposition of such a condition on the approximating spaces. In this article, we discuss and analyze error estimates for Convection- dominated diffusion problems using $\mathrm{H}^{1}$-Galerkin mixed finite element method, along with the method of characteristics. Optimal order of convergence has been achieved for the error estimates of a two-step Euler backward difference scheme.
\end{abstract}

Keywords: $\mathrm{H}^{1}$-Galerkin Mixed Finite Element Method; Characteristics Method; LBB Condition; Optimal Error Estimates; and Euler Backward Difference Scheme

\section{Introduction}

The convection-dominated diffusion problems have been treated heavily using finite element methods [1-4]. Mixed finite element method has been proposed by Douglas et al. [5]. But these methods need to satisfy the Ladyzhenskaya-Babuska-Brezzi (LBB), consistency condition [6-9], on the approximating spaces which restrict the choice of the finite element spaces. In this case it is a special case of those defined by Raviart and Thomas [10]. Pani [11] has proposed and analyzed an $\mathrm{H}^{1}$-Galerkin mixed finite element method which is not restrictive in the sense that the approximating finite element spaces don't need to satisfy the LBB condition. Further, piecewise linear polynomials can be considered for the approximating spaces. Recently, an $\mathrm{H}^{1}$-Galerkin mixed finite element method has been discussed for a class of second order Schrödinger equation by LIU et al. [12].

Moreover, in convection dominated problems, standard upwind finite difference methods are used for problems which artificially smear fronts with excessive numerical dispersion and produce solutions that depend strongly on the orientation of the difference grids relative to the streamlines of flow. Other standard techniques without unwinding produce unacceptable oscillations in the approximations. These difficulties can be reduced substantially by using the Modified Method of Characteristics (MMOC). This procedure was introduced and ana- lyzed for a single parabolic equation by Douglas [13] using backward single-step in the direction of characteristic. Also, this procedure using two-step in the direction of characteristic has been analyzed by Ewing and Russell [14], and then extended by Russell [15] to nonlinear coupled systems in two and three spatial dimensions. The $\mathrm{H}^{1}$-Galerkin mixed finite element along with the method of characteristics has been applied to the convection dominated diffusion problems with a single step backward Euler in the characteristic direction by Mohamed Ali [16].

In this paper, to utilize the above advantages for the convection dominated diffusion problems, we shall propose an H1-Galerkin mixed finite element method combined with the method of characteristics, and examine the rate of convergence for a Two-Step Euler backward difference scheme.

\section{Variational Formulation}

\subsection{Consider the Convection-Dominated Diffusion Problem}

$$
\begin{aligned}
& c(x) \frac{\partial u}{\partial t}+b(x) \frac{\partial u}{\partial x}-\frac{\partial}{\partial x}\left(a(x) \frac{\partial u}{\partial x}\right)=f(x, t), \\
& (x, t) \in[0,1] \times J \\
& u(0, t)=u(1, t)=0, u(x, 0)=u_{0}(x), \quad x \in[0,1] .
\end{aligned}
$$

Assume that $a \geq a_{0}>0$ and $c(x) \geq c_{0}>0$, and the 
coefficients are smooth, where $J=(0,1]$. The above problem arises in many applications involving diffusion processes such as heat flow in a moving material, transport of pollutants in lakes or channels, subsurface hydrology and miscible displacement in porous media.

Setting $v=a(x) u_{x}$, Equation (1) can be rewritten as

$$
u_{t}+\beta(x) v-\gamma v_{x}=\gamma f(x, t),
$$

where $\beta(x)=b(x) / a(x) c(x)$ and $\gamma(x)=1 / c(x)$.

Let $H_{0}^{1}(I)=\left\{\varphi \in H^{1}(I): \varphi(0)=\varphi(1)=0\right\}$. We now state the weak formulation as follows: Find a pair $\{u, v\}: J \rightarrow H_{0}^{1} \times H^{1}$ such that

(a) $\left(a u_{x}, w_{x}\right)=\left(v, w_{x}\right), w \in H_{0}^{1}(I)$

(b) $\left(u_{t}, z_{x}\right)+\left(\beta v, z_{x}\right)-\left(\gamma v_{x}, z_{x}\right)=\left(\gamma f, z_{x}\right), z \in H^{1}(I)$.

Integrating by parts the first two terms of (3.b) we have

$$
\begin{aligned}
& -\left(u_{t x}, z\right)-\left(\beta v_{x}, z\right)-\left(\beta^{\prime} v, z\right)-\left(\gamma v_{x}, z_{x}\right) \\
& =\left(\gamma f, z_{x}\right)-\beta_{1} v(1) z(1)+\beta_{0} v(0) z(0),
\end{aligned}
$$

and hence with $\alpha(x)=1 / a(x)$, we obtain

$$
\begin{aligned}
& \left(\alpha v_{t}+\beta v_{x}, z\right)+\left(\beta^{\prime} v, z\right)+\left(\gamma v_{x}, z_{x}\right) \\
& =-\left(\gamma f, z_{x}\right)+\beta_{1} v(1) z(1)-\beta_{0} v(0) z(0), z \in H^{1}(I) .
\end{aligned}
$$

Here, $\beta_{1}=\beta(1)$ and $\beta_{0}=\beta(0)$. We shall use the standard Galerkin method to solve equation (3.a), and the characteristics method combined with the Galerkin procedure to approximate (4).

With $\psi(x)=\sqrt{\alpha(x)^{2}+\beta(x)^{2}}$, let $\tau=\tau(x)$ denote the characteristic direction associated with the operator $\alpha v_{t}+\beta v_{x}$, where

$$
\frac{\partial}{\partial \tau(x)}=\frac{\alpha(x)}{\psi(x)} \cdot \frac{\partial}{\partial t}+\frac{\beta(x)}{\psi(x)} \cdot \frac{\partial}{\partial x} .
$$

Therefore, the term $\left(\alpha v_{t}+\beta v_{x}, z\right)$ in (4) may be replaced by $\left(\psi(x) \frac{\partial v}{\partial \tau}, z\right)$. Defining the bilinear form

$$
A(v, z)=\left(\gamma v_{x}, z_{x}\right)+\left(\beta^{\prime} v, z\right), \quad z \in H^{1}(I) .
$$

Then Equation (4) can be rewritten as:

$$
\begin{aligned}
& \left(\psi(x) \frac{\partial v}{\partial \tau(x)}, z\right)+A(v, z) \\
& =-\left(\gamma f, z_{x}\right)+\beta_{1} v(1) z(1)-\beta_{0} v(0) z(0), z \in H^{1}(I) .
\end{aligned}
$$

In our error analysis we shall use standard Sobolev spaces $H^{m}(I)$ with norm $\|\cdot\|_{m}$. For simplicity, we write $H^{m}(I)$ as $H^{m}$.

\section{Second-Order Backward Euler Method}

Let $V_{h}$ and $W_{h}$ be the finite dimensional subspaces of
$H^{1}(I)$ and $H_{0}^{1}(I)$, respectively. Assume that $V_{h}$ and $W_{h}$ satisfy the following approximation properties, for $k>0$ and $r>0$ integers,

$$
\begin{gathered}
\inf _{v_{h} \in V_{h}}\left\{\left\|v-v_{h}\right\|+h\left\|v-v_{h}\right\|_{1}\right\} \leq C h^{k+1}\|v\|, \quad v \in H_{0}^{1} \cap H^{k+1} ; \\
\inf _{w_{h} \in W_{h}}\left\{\left\|w-w_{h}\right\|_{L^{p}(I)}+h\left\|w-w_{h}\right\|_{W^{1, p}(I)}\right\} \leq C h^{r+1}\|w\|_{W^{r+1, p}(I)}, \\
w \in H^{r+1} .
\end{gathered}
$$

We shall approximate (5) using the second-order backward Euler approximation method, using the formula

$$
\frac{\partial u^{n}}{\partial t} \cong \frac{\frac{3}{2} u^{n}-2 u^{n-1}+\frac{1}{2} u^{n-2}}{\Delta t} .
$$

For the approximation along the characteristic direction, we define

$$
\begin{gathered}
\bar{x}=x-\frac{\beta(x)}{\alpha(x)} \Delta t, \quad \bar{x}=x-2 \frac{\beta(x)}{\alpha(x)} \Delta t, \\
\bar{u}^{n-1}=u\left(\bar{x}, t^{n-1}\right)=u^{n-1}(\bar{x}),
\end{gathered}
$$

and

$$
\stackrel{u}{=n-1}^{n} u\left(\bar{x}, t^{n-2}\right)=u^{n-2}(\overline{\bar{x}}) .
$$

Then, the time derivative along the characteristic direction is approximated by

$$
\psi \frac{\partial v^{n}}{\partial \tau} \cong \psi \frac{\frac{3}{2} v^{n}-2 \bar{v}^{n-1}+\frac{1}{2} v^{n-2}}{\Delta t\left[1+\frac{\beta^{2}}{\alpha^{2}}\right]^{\frac{1}{2}}} \cong \alpha \frac{\frac{3}{2} v^{n}-2 \bar{v}^{n-1}+\frac{1}{2} v^{=n-2}}{\Delta t}
$$

Then, the Galerkin procedure is to determine a pair $\{U, V\}:\left\{t_{0}, t_{1}, \cdots, t_{N}\right\} \rightarrow W_{h} \times V_{h}$ such that

(a) $\left(a U_{x}^{n}, w_{x}\right)=\left(V^{n}, w_{x}\right), w \in W_{h}$

(b) $\left.\begin{array}{rl} & \left.\alpha \frac{\frac{3}{2} V^{n}-2 \bar{V}^{n-1}+\frac{1}{2} \overline{\bar{V}}^{n-2}}{\Delta t}, z\right)+A_{\lambda}\left(V^{n}, z\right) \\ = & -\left(\gamma f^{n}, z_{x}\right)+\beta_{1} V^{n}(1) z(1)-\beta_{0} V^{n}(0) z(0), z \in V_{h}\end{array}\right\}$

where $A_{\lambda}(\varphi, \psi)=A(\varphi, \psi)+\lambda(\varphi, \psi)$. Here, $\lambda$ is chosen large enough to ensure the coercivity of $A_{\lambda}$, that is there exists $\alpha_{0}>0$ such that

$$
A_{\lambda}(\varphi, \varphi) \geq \alpha_{0}\|\varphi\|_{1}^{2}
$$

Following Wheeler [17], we now define elliptic projections $\tilde{U} \in W_{h}$ and $\tilde{V} \in V_{h}$ of $u$ and $v$ respecttively, through 


$$
\left.\begin{array}{l}
\text { (a) }\left(a\left(u_{x}-\tilde{U}_{x}\right), w_{x}\right)=0, w \in W_{h} \\
\text { (b) } A_{\lambda}(v-\tilde{V}, z)=0, z \in V_{h}
\end{array}\right\}
$$

Letting $\rho=u-\tilde{U}$ and $\eta=v-\tilde{V}$, it is now quite standard to obtain the following estimates, for $1 \leq p \leq \infty$,

$$
\left.\begin{array}{l}
\text { (a) }\|\rho\|_{j, p}+\left\|\rho_{t}\right\|_{j, p} \leq C h^{r+1-j}\left\{\|u\|_{r+1, p}+\left\|u_{t}\right\|_{r+1, p}\right\}, j=0,1 ; \\
\text { (b) }\|\eta\|_{j, p}+\left\|\eta_{t}\right\|_{j, p} \leq C h^{k+1-j}\left\{\|v\|_{k+1, p}+\left\|v_{t}\right\|_{k+1, p}\right\}, j=0,1 .
\end{array}\right\}
$$

Following Pani and Anderssen [18], we have the following super convergence results at the knot points $x=0,1$

$$
\text { (c) }|\eta(1)|+|\eta(0)| \leq C h^{2 k}\|v\|_{k+1} \text {. }
$$

\section{A Priori Error Estimates for Double-Step Backward Euler Method}

Let

$$
u-U=(u-\tilde{U})+(\tilde{U}-U)=\rho+\xi
$$

and

$$
v-V=(v-\tilde{V})+(\tilde{V}-V)=\eta+\zeta .
$$

Now, subtract (7.a) from (3.a) then use (8.a) to have the following error equation in $\xi$

$$
\left(a \xi_{x}^{n}, w_{x}\right)=\left(\eta^{n}+\zeta^{n}, w_{x}\right), w \in W_{h} .
$$

Below we state the following lemma for our subsequent use (the proof of this lemma is given in Mohamed Ali [16], page 46).

\section{Lemma 4.1}

Let $U^{n}$ be an approximate solution satisfying (7.a) and $\tilde{U}^{n}$ be the elliptic projection of $u$ defined through (8.a). Then $\xi=\tilde{U}-U$ satisfies the following estimates,

$$
\left\|\xi^{n}\right\|_{1} \leq C\left[h^{k+1}\|v\|_{k+1}+\left\|\zeta^{n}\right\|\right] .
$$

In the remaining part of this section we shall assume that the coefficients $\beta$ and $\alpha$ are bounded, and $a(x)$ is bounded below by a positive constant, then

$$
\left|\frac{\beta(x)}{\alpha(x)}\right|+\left|\frac{\mathrm{d}}{\mathrm{d} x}\left(\frac{\beta(x)}{\alpha(x)}\right)\right| \leq K .
$$

Now define the elliptic projection $\tilde{V}$ of $v$ through (8.b) and the estimates (9.b) and (9.c) are hold.

The starting procedure for (7.b) will be required to satisfy

$$
\left\|\zeta^{1}\right\|+(\Delta t)^{\frac{1}{2}}\left\|\zeta^{1}\right\|_{1} \leq K\left(h^{k+1}+(\Delta t)^{2}\right)
$$

This can be achieved by using a first-order method on a time step smaller than $\Delta t$, followed by several uses of a multi-step procedure on somewhat larger steps until time $\Delta t$ is reached, for details, see Ewing and Russell [14].

We now state and proof the following theorem.

Theorem 4.1

Suppose that (12) hold. Then the error $\zeta$ of procedure (7.b) satisfies

$$
\begin{aligned}
\max _{0 \leq n \leq N}\left\|\zeta^{n}\right\| & \leq K\left(\|v\|_{L^{\infty}\left(0, T ; H^{k+1}\right)}+\left\|v_{t}\right\|_{L^{2}\left(0, T ; H^{1}\right)}\right) h^{k+1} \\
& +K\left\|\frac{\partial^{3} v}{\partial \tau^{3}}\right\|_{L^{2}\left(0, T ; L^{2}\right)}(\Delta t)^{2} .
\end{aligned}
$$

Proof

Choose $z=\zeta^{n}$ in (7.b) and note that $\zeta^{0}=0$. Then, the error equation in $\zeta$ can be written as

$$
\begin{aligned}
& \left(\alpha \frac{\frac{3}{2} \zeta^{n}-2 \bar{\zeta}^{n-1}+\frac{1}{2} \bar{\zeta}^{n-2}}{\Delta t}, \zeta^{n}\right)+A_{\lambda}\left(\zeta^{n}, \zeta^{n}\right) \\
& =\lambda\left(\zeta^{n}+\eta^{n}, \zeta^{n}\right)+\left(\psi \frac{\partial v^{n}}{\partial \tau}-\alpha \frac{\frac{3}{2} v^{n}-2 \bar{v}^{n-1}+\frac{1}{2} v^{n-2}}{\Delta t}, \zeta^{n}\right) \\
& +\beta_{1} z(1)\left(\eta^{n}(1)+\zeta^{n}(1)\right)+\left(\alpha \frac{\frac{3}{2} \eta^{n}-2 \bar{\eta}^{n-1}+\frac{1}{2} \bar{\eta}^{n-2}}{\Delta t}, \zeta^{n}\right) \\
& -\beta_{0} z(0)\left(\eta^{n}(0)+\zeta^{n}(0)\right)
\end{aligned}
$$

So that

$$
\begin{aligned}
& \left(\alpha \frac{\frac{3}{2} \zeta^{n}-2 \zeta^{n-1}+\frac{1}{2} \zeta^{n-2}}{\Delta t}, \zeta^{n}\right)+A_{\lambda}\left(\zeta^{n}, \zeta^{n}\right) \\
& =\lambda\left(\zeta^{n}+\eta^{n}, \zeta^{n}\right)+\left(\psi \frac{\partial v^{n}}{\partial \tau}-\alpha \frac{\frac{3}{2} \nu^{n}-2 \bar{v}^{n-1}+\frac{1}{2} v}{\Delta t}, \zeta^{n-2}\right) \\
& +2\left(\alpha \frac{\bar{\zeta}^{n-1}-\zeta^{n-1}}{\Delta t}, \zeta^{n}\right)+\frac{1}{2}\left(\alpha \frac{\zeta^{n-2}-\bar{\zeta}^{n-2}}{\Delta t}, \zeta^{n}\right) \\
& +2\left(\alpha \frac{\eta^{n}-\eta^{n-1}}{\Delta t}, \zeta^{n}\right)+2\left(\alpha \frac{\eta^{n-1}-\bar{\eta}^{n-1}}{\Delta t}, \zeta^{n}\right) \\
& \quad-\left(\alpha \frac{\eta^{n}-\eta^{n-2}}{2 \Delta t}, \zeta^{n}\right)+\frac{1}{2} 2\left(\alpha \frac{\eta^{n-2}-\eta^{n-2}}{\Delta t}, \zeta^{n}\right) \\
& +\left[\beta_{1} z(1)\left(\eta^{n}(1)+\zeta^{n}(1)\right)-\beta_{0} z(0)\left(\eta^{n}(0)+\zeta^{n}(0)\right)\right] \\
& =\sum_{i=1}^{9}\left(F_{i}, \zeta^{n}\right) \equiv\left(F^{n}, \zeta^{n}\right),
\end{aligned}
$$


To treat the left hand side of (13), we use the following stability lemma.

Lemma 4.2

If (12) and (13) hold, then for $2 \leq l \leq N$,

$$
\begin{aligned}
\left(\alpha \zeta^{l}, \zeta^{l}\right)+ & \frac{4}{3} \sum_{n=2}^{l} A_{\lambda}\left(\zeta^{n}, \zeta^{n}\right) \Delta t \leq \frac{1}{3}\left(\alpha \zeta^{l-1}, \zeta^{l-1}\right) \\
& +\frac{4}{3} \sum_{n=2}^{l}\left(F^{n}, 2 \zeta^{l}-\zeta^{l-1}\right) \Delta t+K\left(h^{2 k+2}+(\Delta t)^{4}\right)
\end{aligned}
$$

For proof of this lemma, refer to R.E Ewing and Russell [14].

By the lemma 4.2, theorem 4.1, will be proved if we can handle the terms on the right-hand side of (14). The first term can be ignored because of the coefficient $\frac{1}{3}$; a recursion argument would show that it could be removed if the other terms were multiplied by $1+\frac{1}{3}+\frac{1}{3^{2}}+\cdots=\frac{3}{2}$. For the second term, expressions of the forms $\varepsilon \sum\left\|\alpha \zeta^{n}\right\|_{1}^{2} \Delta t$ and $K \sum\left\|\alpha \zeta^{n}\right\|^{2} \Delta t$ can be respectively hidden on the left-hand side of (13) and eliminated by the discrete Gronwall's lemma. Hence, using (9.b), the first term on the right hand side o (13) can be handled as:

$$
\begin{aligned}
& \text { I.e., } \quad\left|\lambda\left(\zeta^{n}+\eta^{n}, \zeta^{n}\right)\right| \leq \varepsilon\left\|\zeta^{n}\right\|_{1}^{2}+K h^{2 k+2}\|v\|_{k+1}^{2} . \\
& \quad \sum_{n=2}^{l}\left\|F_{1}^{n}\right\|^{2} \Delta t \leq K h^{2 k+2}\|v\|_{L^{\infty}\left(0, T ; H^{k+1}\right)}^{2}
\end{aligned}
$$

and as in Ewing and Russell [14], the following terms on the right-hand side of (13) is bounded by

$$
\begin{aligned}
& \sum_{n=2}^{l}\left\|F_{2}^{n}\right\|^{2} \Delta t \leq K\left\|\frac{\partial^{3} v}{\partial \tau^{3}}\right\|_{L^{2}\left(0, T ; L^{2}\right)}^{2}(\Delta t)^{4}, \\
& \sum_{n=2}^{l}\left\|F_{3}^{n}\right\|^{2} \Delta t \leq \varepsilon \sum_{n=2}^{l}\left\|\zeta^{n-1}\right\|_{1}^{2} \Delta t, \\
& \sum_{n=2}^{l}\left\|F_{4}^{n}\right\|^{2} \Delta t \leq \varepsilon \sum_{n=2}^{l}\left\|\zeta^{n-2}\right\|^{2} \Delta t, \\
& \sum_{n=2}^{l}\left\|F_{5}^{n}\right\|^{2} \Delta t \leq K h^{2 k+2}\left\|u_{t}\right\|_{L^{2}\left(0, T ; H^{k}\right)}^{2}, \\
& \sum_{n=2}^{l}\left\|F_{6}^{n}\right\|^{2} \Delta t \leq K h^{2 k+2}\|v\|_{L^{\infty}}^{2}\left(0, T ; H^{k+1}\right), \\
& \sum_{n=2}^{l}\left\|F_{7}^{n}\right\|^{2} \Delta t \leq K h^{2 k+2}\left\|u_{t}\right\|_{L^{2}\left(0, T ; H^{k}\right)}^{2}, \\
& \sum_{n=2}^{l}\left\|F_{8}^{n}\right\|^{2} \Delta t \leq K h^{2 k+2}\|v\|_{L^{\infty}\left(0, T ; H^{k+1}\right)}^{2},
\end{aligned}
$$

and as in Mohamed Ali [16], the last term is bounded by

$$
\sum_{n=2}^{l}\left\|F_{9}^{n}\right\|^{2} \Delta t \leq K h^{4 k}\|V\|_{L^{\infty}\left(0, T ; H^{k+1}\right)}^{2}
$$

Inserting these results in (14) completes the proof of the theorem.

\section{Conclusion}

The computational process of the classical mixed finite element methods faces some difficulties while choosing the finite element spaces due to the restrictive LBB condition which is a must for such methods. In this paper, the application of this condition has been avoided by using the $\mathrm{H}^{1}$-Galerkin mixed finite element method. For more accurate and fast results, we have used the modified characteristics method for two-step backward euler time discretization. This allows choosing the finite element spaces freely and maintaining the optimality of the order of convergence of the analysis. This problem has a wide range of applications in the real life such as in the transport of air and water pollutants, in oil reservoir flow, in the modeling of semiconductor and so forth.

\section{REFERENCES}

[1] A. C. Galeao, R. C. Almeida, S. M. C. Malta and A. F. D. Loula, "Finite Element Analysis of Convection-Dominated Reaction-Diffusion Problems," Applied Numerical Mathematics, Vol. 48, No. 2, 2004, pp. 205-222. doi:10.1016/j.apnum.2003.10.002

[2] M. El-Amrani and M. Seaid, “A Finite Element Modified Method of Characteristics for Convection Heat Transport," Numerical Methods for Partial Differential Equations, Vol. 24, No. 3, 2008, pp. 776-798. doi:10.1002/num.20288

[3] F. Freiberger, "Numerical Solution of Convection- Dominated Problems Using Isogeometric Analysis,” Institute fur Mathematik, TU Clausthal, Clausthal-Zellerfeld, 2010.

[4] J. Volker and N. Julia, "Error Analysis of the SUPG Finite Element Discretization of Evolutionary ConvectionDiffusion-Reaction Equations," SIAM Journal on $\mathrm{Nu}$ merical Analysis, Vol. 49, No. 3, 2011, pp. 1149-1176.

[5] J. Douglas Jr., R. E. Ewing and M. F. Wheeler, "A TimeDiscretization Procedure for a Mixed Finite Element Approximation of Miscible Displacement in Porous Media," ESAIM: Mathematical Modelling and Numerical Analysis: Modélisation Mathématique et Analyse Numérique, Vol. 17, No. 3, 1983, pp. 249-265.

[6] I. Babuska, "The Finite Element Method with Lagrange Multipliers,” Numerische Mathematik, Vol. 20, No. 3, 1973, pp. 179-192. doi:10.1007/BF01436561

[7] F. Brezzi, "On the Existence, Uniqueness and Approximation of Saddle Point Problems Arising from Lagrange Multipliers”, ESAIM: Mathematical Modelling and $\mathrm{Nu}$ merical Analysis: Modélisation Mathématique et Analyse Numérique, Vol. 8, No. 2, 1974, pp. 129-151. 
[8] O. A. Ladyzhenskaya, "The Mathematical Theory of Viscous Incomplete Flows,” Gordon and Breach, London, 1969.

[9] T. Arbogast and M. F. Wheeler, "A CharacteristicsMixed Finite Element Method for Advection Dominated Transport Problems," SIAM Journal on Numerical Analysis, Vol. 32, No. 2, 1995, pp. 404-424. doi:10.1137/0732017

[10] P. A. Raviart and J. M. Thomas, "A Mixed Finite Element Method for Second Order Elliptic Problems,” Mathematical Aspects of the Finite Element Method, Lecture Notes in Mathematics, Vol. 66, 1977, pp. 292-315. doi:10.1007/BFb0064470

[11] A. K. Pani, "H1-Galerkin Mixed Finite Element Method for Parabolic Equations," SIAM Journal on Numerical Analysis, Vol. 35, No. 2, 1998, pp. 712-727. doi:10.1137/S0036142995280808

[12] Y. Liu, H. Li and J.-F. Wang, "Error Estimates of H1-Galerkin Mixed Finite Element Method for Schrödinger Equation,” Applied Mathematics-A Journal of Chinese Universities, Vol. 24, No. 1, 2009, pp. 83-89. doi:10.1007/s11766-009-1782-3

[13] J. Douglas Jr. and T. F. Russell, "Numerical Methods for Convection-Diffusion Problems Based on Combining the method of Characteristics with Finite Element or Finite
Difference Procedures,” SIAM Journal on Numerical Analysis, Vol. 19, 1982, pp. 871-885. doi:10.1137/0719063

[14] Ewing, R. E. and T. F. Russell, "Multistep Galerkin Methods along Characteristics for Convection-Diffusion Problems,” In: R. Vichnevetsky and R. S. Stepkman, Eds., Advances in Computer Methods for Partial Differential Equations, IMACS, Rutgers University, New Brunswick, 1981, pp. 28-36.

[15] T. F. Russell, “Time-Stepping along Characteristics with Incomplete Iteration for a Galerkin Approximation of Miscible Displacement in Porous Media," SIAM Journal on Numerical Analysis, Vol. 22, No. 5, 1985, pp. 9701013. doi:10.1137/0722059

[16] M. A. Mohamed Ali, “An Error Estimates for Convection Dominated Diffusion Problems," Journal of Natural Sciences and Mathematics, Vol. 6, No. 1, 2013, pp. 41-53.

[17] M. F. Wheeler, "Apriori L2-Error Estimates for Galerkin Approximations to Parabolic Differential Equations," SIAM Journal on Numerical Analysis, Vol. 10, No. 4, 1973, pp. 723-749. doi:10.1137/0710062

[18] A. K. Pani and R. S. Anderssen, "Finite Element Methods for Identification of Parameters in Parabolic Problems," Proceedings of the Centre for Mathematics and Its Applications, Vol. 31, 1992, pp. 208-221. 